Short Communication

Animal Genetics

\title{
Establishment of rapid and non-invasive protocols to identify B-carrying individuals of Psalidodon paranae
}

Caio Augusto Gomes Goes ${ }^{1}$ (D), Dúlio Mazzoni Zerbinato de Andrade Silva ${ }^{2}$, Ricardo Utsunomia ${ }^{3}$, George Shigueki Yasui ${ }^{4}$, Roberto Ferreira Artoni ${ }^{5}$, Fausto Foresti ${ }^{2}$ and Fábio Porto-Foresti ${ }^{1}$

'Universidade Estadual Paulista "Júlio de Mesquita Filho" (UNESP), Faculdade de Ciências, Bauru, SP, Brazil

${ }^{2}$ Universidade Estadual Paulista "Júlio de Mesquita Filho" (UNESP), Instituto de Biociências, Botucatu, SP, Brazil

${ }^{3}$ Universidade Federal Rural do Rio de Janeiro, Instituto de Ciências Biológicas e da Saúde, ICBS, Seropédica, RJ, Brazil

${ }^{4}$ Centro nacional de Pesquisa e Conservação da Biota Aquática Continental (CEPTA-ICMBIO), Pirassununga, SP, Brazil

${ }^{5}$ Universidade Estadual de Ponta Grossa, Setor de Ciências Biológicas e da Saúde, Ponta Grossa, PR, Brazil

\begin{abstract}
Supernumerary, or B, chromosomes are present in several eukaryotes, including characid fish of the genus Psalidodon. Notably, Psalidodon paranae carries the most studied B chromosome variant, a macro-B chromosome. The origin of this element was determined to be an isochromosome; however, data regarding its inheritance remain unavailable due to methodological barriers such as the lack of an efficient, non-invasive, and rapid protocol for identifying B-carrying individuals that would enable the design of efficient crossing experiments. Thus, in this study, we primarily aimed was to develop two non-invasive and fast (approximately $2 \mathrm{~h}$ ) methods to identify the presence of $\mathrm{B}$ chromosomes in live specimens of $P$. paranae based on satellite DNA (satDNA) sequences known to be present in this element. The methods include fluorescence in situ hybridization in interphase nuclei and relative gene quantification of satDNAs using quantitative polymerase chain reaction. Our results reveal the efficiency of quickfluorescence in situ hybridization and quantitative polymerase chain reaction for identifying B-carrying individuals using the proposed satDNA sequences and open up new possibilities to study B chromosomes.
\end{abstract}

Keywords: Psalidodon, Neotropical fishes, satellite DNA, qPCR, B chromosomes.

Received: January 22, 2020; Accepted: February 12, 2021.

Supernumerary or B chromosomes are dispensable chromosomes additional to the standard A complement (Jones and Rees, 1982; Camacho et al., 2000). These elements can be found in diverse species (including plants, animals, and fungi) (Camacho et al., 2000; Burt and Trivers, 2006; Ahmad and Martins, 2019), representing approximately 15\% of eukaryotes. In general, B chromosomes do not follow a Mendelian segregation pattern and do not always occur in pairs, which can facilitate inheritance values higher than 0.5 , resulting in transmission advantages, or the so-called "drive" process (Houben, 2017).

In fish, B chromosomes have been reported in approximately 113 species, of which 38 are found in the Neotropical region, such as characid fish of the genus Psalidodon (D'Ambrosio et al., 2017). Remarkably, one can observe a wide variety of B chromosomes in Psalidodon, with

Send correspondence to: Fábio Porto-Foresti. Universidade Estadual Paulista "Júlio de Mesquita Filho" (UNESP), Faculdade de Ciências, Departamento de Ciências Biológicas, Av. Eng. Luiz Edmundo Carrijo Coube, 14-01, 17033-360, Bauru, SP, Brazil. E-mail: fp.foresti@ unesp.br. primary differences in their morphology, ranging from large metacentric chromosomes similar in size to the first pair of chromosomes in P. scabripinnis and P. paranae (Salvador and Moreira-Filho, 1992; Silva et al., 2014) to microchromosomes, in P. bockmanni (Daniel et al., 2012).

During the preceding decades, the large metacentric B chromosome variant, or the macro-B chromosome, observed in seven species of Psalidodon, was analyzed using distinct approaches (Maistro et al., 1992; Salvador and Moreira-Filho, 1992; Moreira-Filho et al., 2001; Torres-Mariano and Morelli, 2008; Silva et al., 2016). These species were originally part of the Astyanax genus, but a recent revision led to their inclusion in the Psalidodon genus (Terán et al., 2020). Fluorescence in situ hybridization (FISH) patterns of repetitive DNAs revealed that this $\mathrm{B}$ chromosome is an isochromosome, of intraspecific origin, of the A complement in P. paranae and P. scabripinnis (Mestriner et al., 2000; Silva et al., 2014). In addition, chromosome painting data revealed a common origin of this macro-B chromosome for several species, with subsequent independent diversification processes (Vicari et al., 2011; Silva et al., 2016; 2017). P. paranae has the highest population frequency of $\mathrm{B}$ chromosomes in the genus, with approximately $40 \%$ of individuals carrying them (Porto-Foresti 
et al., 1997). Furthermore, the accumulated data from nextgeneration sequencing of B-carrying (1B) and non-B-carrying (0B) individuals, obtained through comparative analysis of these two genomes (Silva et al., 2017), show P. paranae to be a model for studying B chromosomes of the genus Psalidodon. Although the origin and evolution of this genomic element were assessed several times using multiple approaches, its inheritance patterns have never been studied, even though such an analysis could assist in understanding the life cycle of this chromosome. Therefore, the development of non-invasive protocols for genotyping B-carrying individuals of $P$. paranae would allow efficient designing of crossing experiments that could uncover myriad mating possibilities for this species.

There is a fundamental need to develop non-invasive methods for identification of fish species that are aimed at preserving individuals without disturbing their integrity. These methods can be applied in many ways, such as measuring the size of internal organs (Näslund, 2014), characterizing immune responses (Matsuura et al., 2017), and identifying the sex of individuals (Utsunomia et al., 2017; 2019). Thus, the standardization of non-invasive protocols to identify $1 \mathrm{~B}$ and $0 \mathrm{~B}$ individuals of $P$. paranae will allow a glimpse into the inheritance patterns of B chromosomes in fish. In this context, the existence of clustered satellite DNA (satDNA) sequences on $\mathrm{B}$ chromosomes of this species provides an opportunity to develop a FISH-based method that uses these repetitive sequences.

In this study, 10 individuals from $P$. paranae (six females and four males) were collected from Cascatinha Stream,

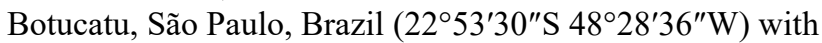
authorization of the relevant organizations (MMA / IBAMA / ICMBio / SISBIO - 18884-1, registered with IBAMA No. 2567470). The procedures, maintenance, and analysis of all fish were performed in compliance with the standards of the Brazilian College of Animal Experimentation. The individuals were deposited in the collection of the Laboratório de Genética de Peixes at UNESP, Bauru, São Paulo, Brazil under voucher LGP 10120 - 10130. Small fragments of fins from each individual were cut and preserved in $96 \%$ alcohol and Carnoy's solution (methanol-acetic acid, 3:1) for laboratory analysis. Then, $15 \mu \mathrm{L}$ of cell suspension was dispensed on the slides and dried at $37{ }^{\circ} \mathrm{C}$ for $30 \mathrm{~min}$. The individuals were then sacrificed by treatment with an overdose of anesthetic in $1 \%$ benzocaine and were later dissected. Next, mitotic chromosomes were obtained from all the sample specimens according to the protocol established by Foresti et al. (1981) to visually confirm the presence of B chromosomes.

The Apasat06-86 satDNA described by Silva et al. (2017) was selected to detect the presence of B chromosomes because the hybridization patterns of the satDNA showed the presence of a large pericentromeric block in the B chromosomes of this species (Silva et al., 2017). FISH experiments in interphase cells were performed according to the protocol established by Utsunomia et al. (2019), with 30 min probe hybridization. The probes were generated as described by Silva et al. (2017), and probe detection was performed using anti-digoxigenin- rhodamine (Roche). Chromosomes and interphase nuclei were counterstained using 4', 6-diamidino-2-phenylindole (Vector Laboratories). The entire process takes approximately $2 \mathrm{~h}$. To verify the efficiency of our methods, approximately 100 interphase nuclei were analyzed.

In addition to the quick-FISH method, a quantitative polymerase chain reaction (qPCR)-based method was performed in parallel, to independently confirm the presence of B chromosomes in the $P$. paranae individuals. The copy numbers of the three satDNA sequences present on $\mathrm{B}$ chromosomes of $P$. paranae were determined using relative quantification (RQ). ApaSat06-86 was used for the quick-FISH experiment, whereas ApaSat20-18 and ApaSat44-21 were tested for their presence exclusively on the B chromosomes in this species using FISH (Silva et al., 2017).

Total DNA was extracted from fin fragments of the individuals using the Wizard Genomics Kit (Promega). qPCR was performed using the StepOne Real-Time PCR System (Life Technologies, Carlsbad, CA, USA). We used the $2^{-\Delta \mathrm{Ct}}$ method (Bel et al., 2011) for RQ, using the single-copy gene hypoxanthine phosphoribosyltransferase (Hprt) as reference. The primers described by Silva et al. (2017) for satDNAs and Utsunomia et al. (2019) for Hprt were used. DNA from three samples each from the $0 \mathrm{~B}$ and $1 \mathrm{~B}$ groups were analyzed simultaneously using three independent replicates for both the target and reference genes. The reactions were performed in a final volume of $10 \mu \mathrm{L}$ with $3 \mathrm{ng}$ of genomic DNA, $5 \mu \mathrm{L}$ of Power SYBR ${ }^{\text {TM }}$ Green PCR Master Mix (Applied Biosystems) and $1 \mu \mathrm{L}$ of each $5 \mu \mathrm{M}$ primer. The cycle conditions were as follows: $95^{\circ} \mathrm{C}$ for $10 \mathrm{~min}$ and 45 cycles of $95^{\circ} \mathrm{C}$ for $15 \mathrm{~s}$ and $60{ }^{\circ} \mathrm{C}$ for $1 \mathrm{~min}$. The specificity of the PCR products was confirmed using dissociation curve analysis. No samples were discarded as the $\mathrm{Ct}$ values showed a consistent pattern. The RQ value is represented as the mean \pm standard error of the mean. Statistical analysis was performed using the ShapiroWilk test to ascertain whether the variables fit a normal distribution, followed by the Student's $t$-test. A summary of the methodology is shown in Figure 1.

FISH in metaphase cells of $P$. paranae revealed the discrete presence of the Apasat06-86 satDNA sequence in the centromeric and telomeric regions of some chromosomes in the A set. In 1B individuals, FISH revealed a large accumulation of this satDNA sequence in the centromeric region of $\mathrm{B}$ chromosomes (Figure 2a-c), corroborating the results obtained by Silva et al. (2017). FISH in interphase cells showed several small signals distributed throughout the cell nuclei in $0 \mathrm{~B}$ individuals, corresponding to the sites observed in the chromosome of the standard set (Figure 2d-f). However, 1B individuals also showed a large and conspicuous mark in their nuclei (Figure 2g-i). Thus, the Apasat06-86 probe was $100 \%$ efficient in identifying $1 \mathrm{~B}$ individuals and eliminated the necessity for a control probe. Our microscopic analysis of $85 \%$ of the cells from each individual presented the expected signal patterns. However, it should be noted that this satDNA sequence is not suitable for qPCR experiments because of its accumulation in chromosomes other than $\mathrm{B}$ chromosomes (Figure 3). 


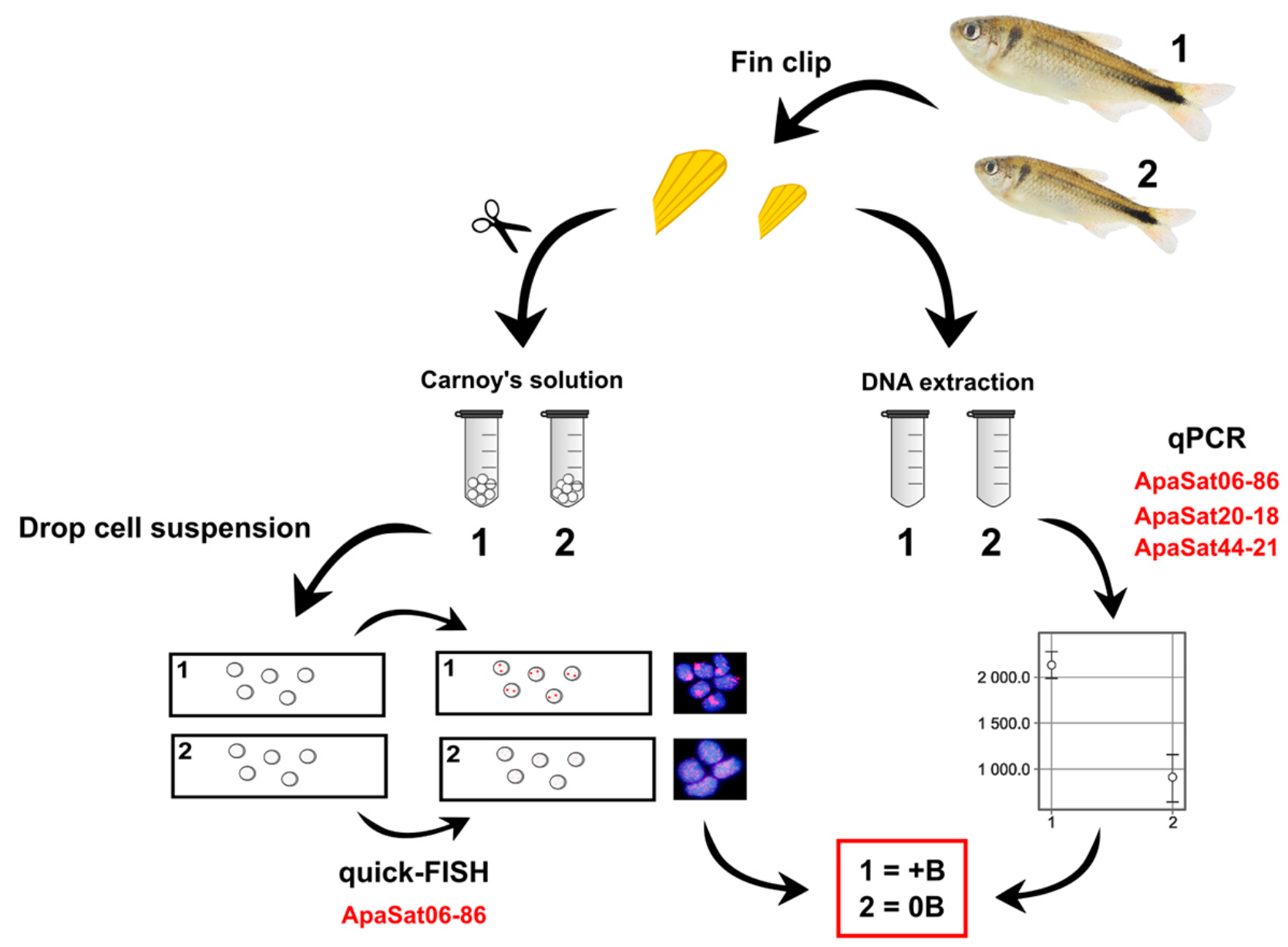

Figure 1 - Schematic summary of the practical steps proposed in this study to identify B-carrying individuals of Psalidodon paranae.

Conventional PCR was performed at intervals of 5,10 , and 15 cycles to verify the amplification of the Apasat06-86 sequence. Except for 5-cycle reactions, samples from both $\mathrm{OB}$ and $1 \mathrm{~B}$ individuals demonstrated the expected banding patterns (Figure 4), confirming that a PCR-based method is not suitable for genotype individuals of $P$. paranae. To overcome this limitation, ApaSat44-21 was tested and confirmed to be an efficient marker, showing a significant difference in genomic abundance when comparing $0 \mathrm{~B}$ and $1 \mathrm{~B}$ individuals (Figure 3 ). FISH mapping of this satDNA confirmed its clustering only in the B chromosomes of $P$. paranae, and not in the genomes of P. fasciatus or P. bockmanni (Silva et al., 2017). qPCR using the other two proposed satellites did not show any significant differences between $0 \mathrm{~B}$ and $1 \mathrm{~B}$ individuals, probably due to their presence in A chromosomes (Figure 3).

The complete satellitome of $P$. paranae was described by Silva et al. (2017), and FISH mapping demonstrated the presence of 15 satDNA sequences in the B chromosomes of this species. In addition, several of these satDNAs are shared by the B chromosome of P. bockmanni and P. fasciatus (Silva et al., 2017), despite the fact that next-generation sequencing data are not available for these two species. In this case, any of the satDNAs shared between the B chromosomes of $P$. paranae and the variants present in $P$. fasciatus and P. bockmanni have the potential to function as markers of B chromosomes. It is possible to replicate the method, which used the Apasat06-86 satDNA, proposed in this study to identify B chromosomes in $P$. paranae utilizing the Apasat20-18 satDNA, which demonstrates the pattern of distribution in P. fasciatus and P. bockmanni (Silva et al., 2017). Despite the potential of this method, B chromosomes are present at a low frequency in $P$. fasciatus and $P$. bockmanni, contrary to what was observed in P. paranae (Porto-Foresti et al., 1997).

Our data demonstrate the practical use of satDNAs as potential biotechnological tools for identifying $P$. paranae individuals with supernumerary, or B, chromosomes. Similar applications have already been demonstrated for Megaleporinus macrocephalus, in which there is an accumulation of satDNA families on the W sex chromosome (Utsunomia et al., 2019). Here, the non-invasive identification of $P$. paranae individuals with B chromosomes could allow the use of these fish for breeding, with the aim of describing the inheritance patterns of supernumerary elements as well as promoting full population studies for detecting the distribution of these elements in natural individuals, including juveniles.

$P$. paranae is an important species for studying B chromosomes in the genus Psalidodon because of the accumulated knowledge regarding its morphology, population 
DAPI
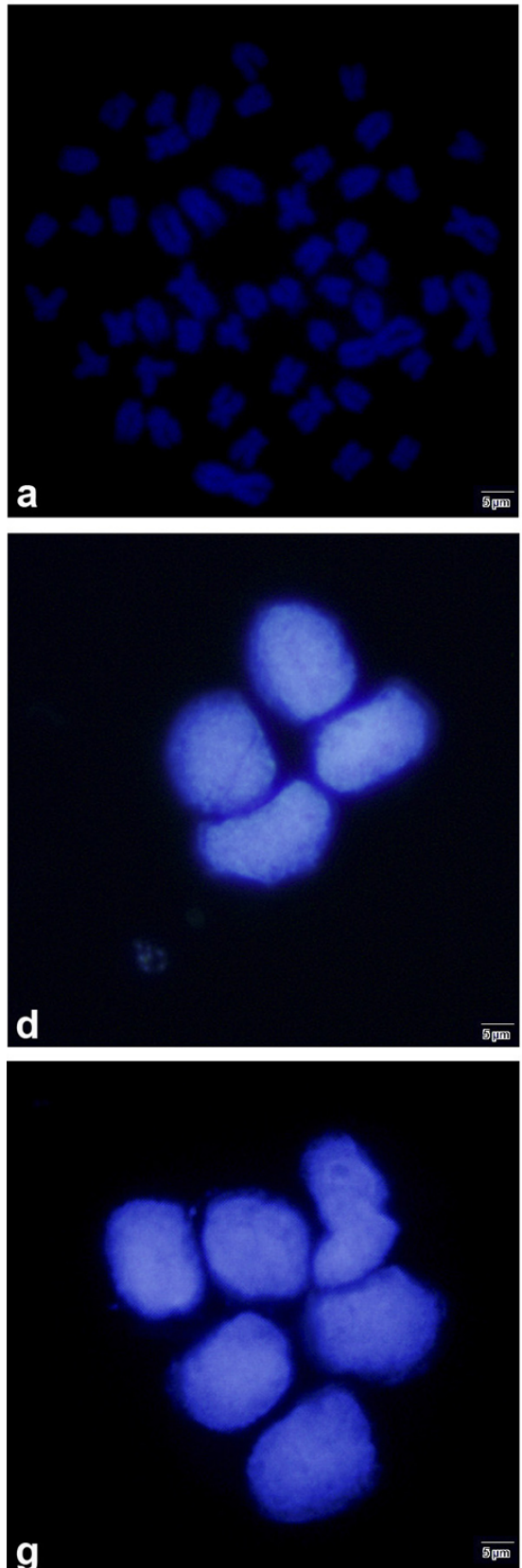

Apasat06-86 probe
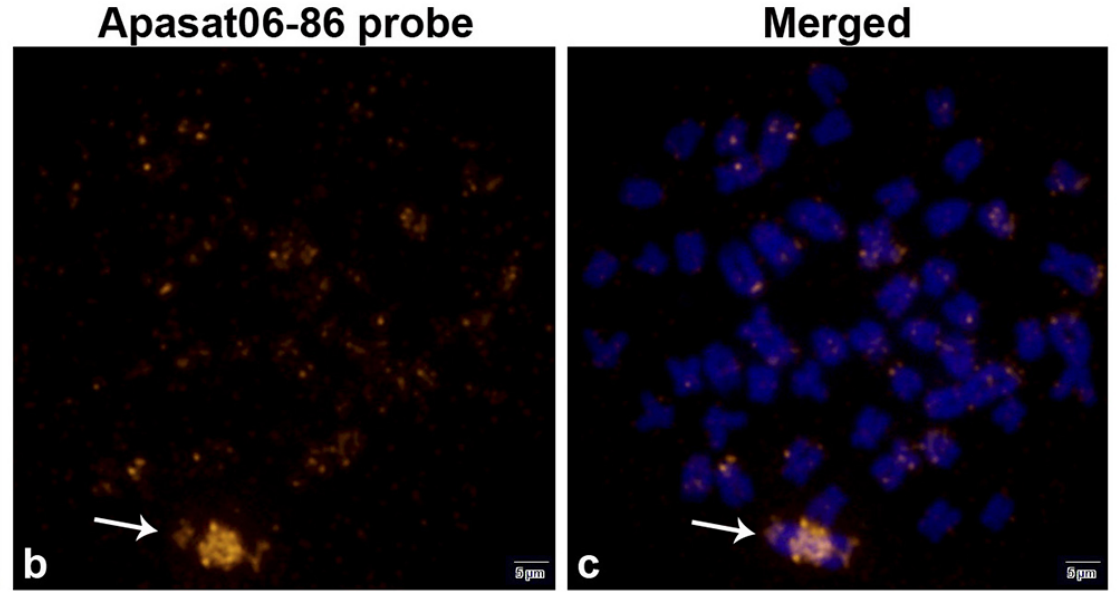

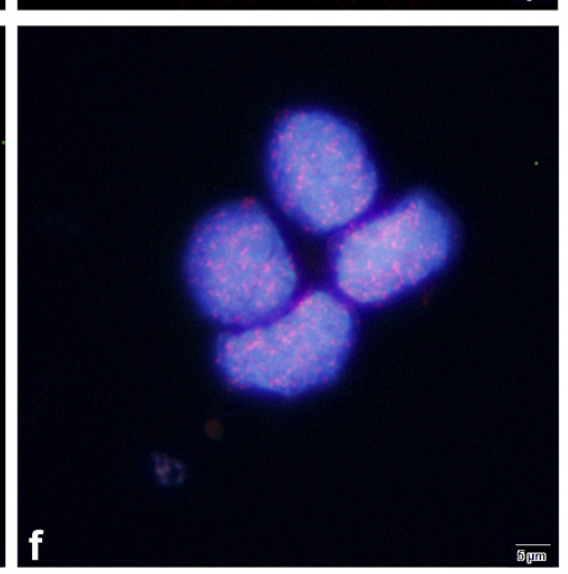

e

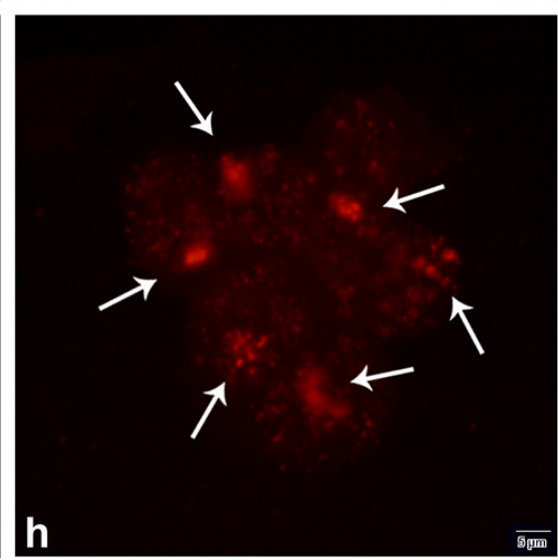

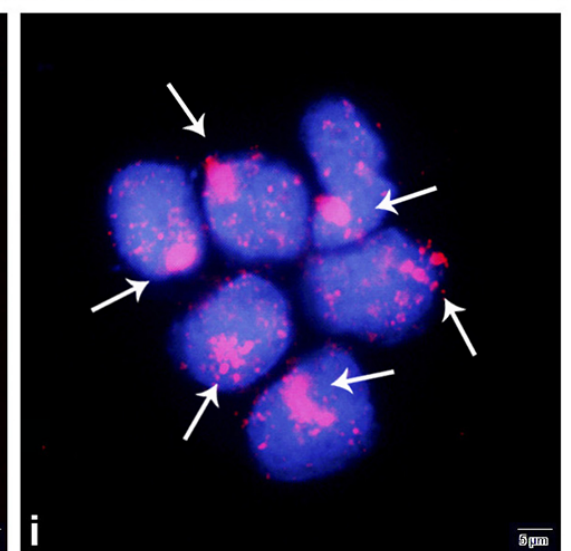

Figure 2 - Signal patterns of signals obtained for the Apasat06-86 satellite after fluorescent in situ hybridization (FISH) in Psalidodon paranae. This sequence displays a conspicuous block corresponding to the B chromosomes of this species shown using arrows, while small signals scattered throughout normal chromosomes can also be seen (b, e, h). It also depicts FISH in the interphase nuclei of non-B-carrying (0B), in which only small-scattered signals are observed (e, f). The same technique performed on B-carrying (1B) individuals illustrates the presence of scattered signals in addition to a large block corresponding to B chromosome $(\mathrm{h}, \mathrm{i})$.

distribution, molecular structure, and genetic content (Maistro et al., 1992; Porto-Foresti et al., 1997; Silva et al., 2014, 2016, 2017). Thus, the use of satDNA sequences to develop faster, more effective, and cheaper identification methods that do not require animal sacrifice is extremely important. This work presents two rapid and effective methods for identifying $P$. paranae individuals carrying $\mathrm{B}$ chromosomes, and the findings may open new possibilities for the study of the evolutionary dynamics of B chromosomes in Psalidodon. 


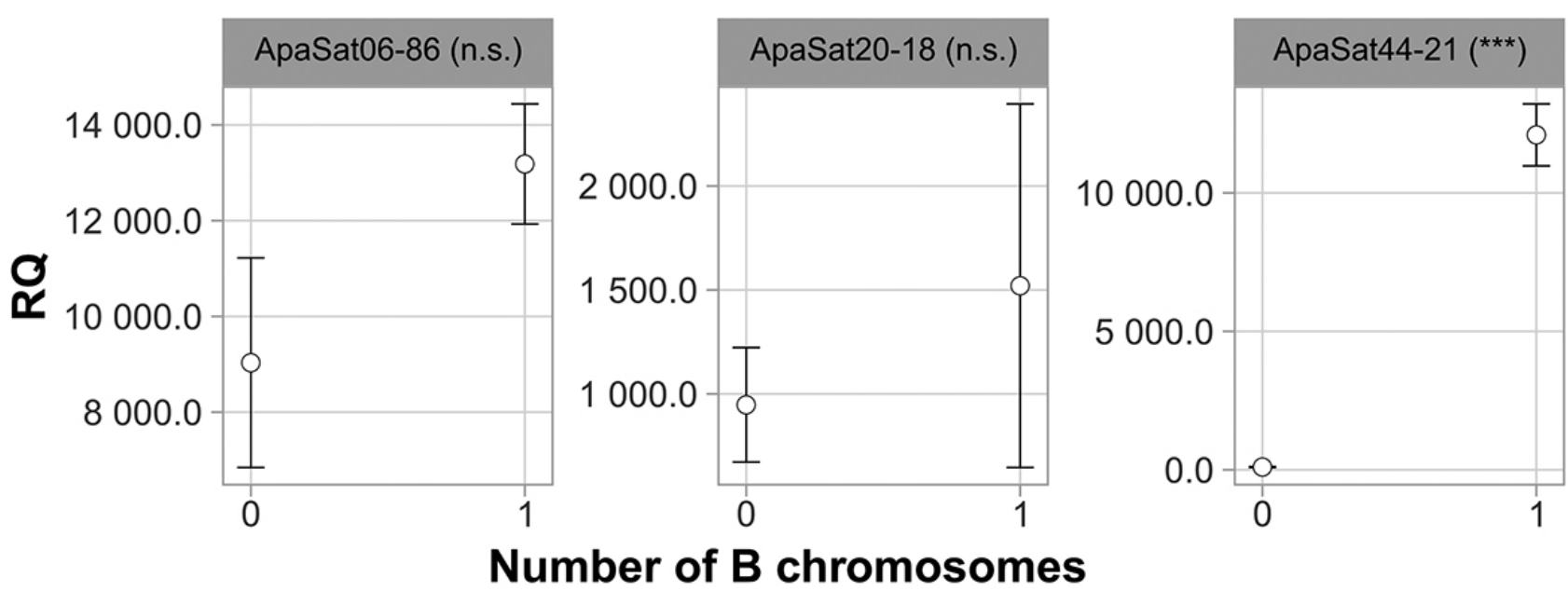

Figure 3 - Relative quantification (RQ) of three satellite DNAs from non-B-carrying (0B) and B-carrying (1B) individuals of Psalidodon paranae. n.s.: $\mathrm{p}>0.05 . * * *: \mathrm{p}<0.001$.

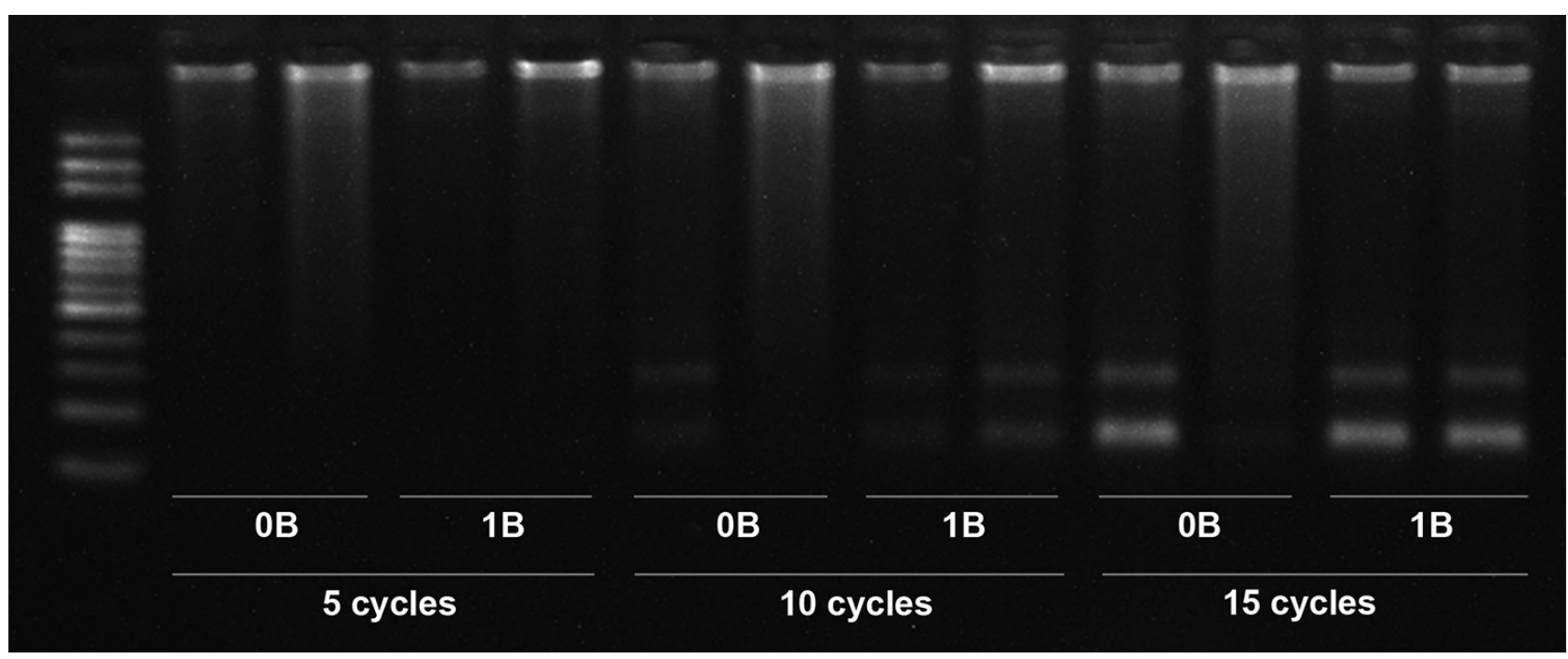

Figure 4 - Agarose gel showing Apasat06-86 sequence amplification. No significant differences were observed in band patterns of non-B-carrying (0B) and B-carrying (1B) individuals after 15 cycles of conventional polymerase chain reaction.

\section{Acknowledgements}

This research was funded by grants from the Fundação de Amparo à Pesquisa do Estado de São Paulo (FAPESP), Conselho Nacional de Desenvolvimento Científico e Tecnológico (CNPq), and Coordenação de Aperfeiçoamento de Pessoal de Nível Superior (CAPES, Brasil - Finance code 100).

\section{Conflict of Interest}

The authors declare that there is no conflict of interest that could be perceived as prejudicial to the impartiality of the reported research.

\section{Authors Contributions}

CAGG, RU and FPF conceived the study and wrote the manuscript; CAGG, DMZAS and FPF conducted the experiments. All authors analyzed the analyzed the data and read and approved the final version.

\section{References}

Ahmad SF and Martins C (2019) The modern view of B chromosomes under the impact of high scale omics analyses. Cells 8:156.

Bel Y, Ferré J and Escriche B (2011) Quantitative real-time PCR with SYBR Green detection to assess gene duplication in insects: study of gene dosage in Drosophila melanogaster (Diptera) and in Ostrinia nubilalis (Lepidoptera). BMC Res Notes 4:84

Burt A and Trivers R (2006) Genes in conflict: the biology of selfish genetic elements. Harvard University Press, Cambridge, 632 pp.

Camacho JPM, Sharbel TF and Beukeboom LW (2000) B-chromosome evolution. Phil Trans R Soc Lond B 355:163-178.

Daniel SN, Hashimoto DT, Pansonato-Alves JC, Foresti F and PortoForesti F (2012) Cytogenetic characterization of distinct B chromosomes in a population of the fish Astyanax bockmanni (Teleostei, Characiformes). Caryologia 65:229-233.

D'Ambrosio U, Alonso-Lifante MP, Barros K, Kovař́k A, Xaxars GM and Garcia S (2017) B-chrom: A database on B-chromosomes of plants, animals and fungi. New Phytol 216:635-642. 
Foresti F, Almeida-Toledo LF and Toledo-Filho SA (1981) Polymorphic nature of nucleolus organizer regions in fishes. Cytogenet Genome Res 31:137-144.

Houben A (2017) B chromosomes-a matter of chromosome drive. Front Plant Sci 8:210.

Jones RN and Rees H (1982) Transmission and drive involving parasitic B chromosomes. Genes 9:388.

Maistro EL, Foresti F, Oliveira C and Almeida-Toledo LF (1992) Occurrence of macro B chromosomes in Astyanax scabripinnis paranae (Pisces, Characiformes, Characidae). Genetica 87:101-106.

Matsuura Y, Takaoka N, Miyazawa R and Nakanishi T (2017) A simple and non-invasive method for analyzing local immune responses in vivo using fish fin. Dev Comp Immunol 74:136143.

Mestriner CA, Galetti Jr PM, Valentini SR, Ruiz IRG, Abel LDS, Moreira-Filho O and Camacho JPM (2000) Structural and functional evidence that a $\mathrm{B}$ chromosome in the characid fish Astyanax scabripinnis is an isochromosome. Heredity 85:1-9.

Moreira-Filho O, Fenocchio AS, Pastori MC and Bertollo LAC (2001) Occurrence of a metacentric macrochromosome B in different species of the genus Astyanax (Pisces, Characidae, Tetragonopterinae). Cytologia 66:59-64

Näslund J (2014) A simple non-invasive method for measuring gross brain size in small live fish with semi-transparent heads. PeerJ 2:e586.

Porto-Foresti F, Oliveira C, Maistro EL and Foresti F (1997) Estimated frequency of B-chromosomes and population density of Astyanax scabripinnis paranae in a small stream. Braz $\mathrm{J}$ Genet 20:377-380

Salvador LB and Moreira-Filho O (1992) B chromosomes in Astyanax scabripinnis (Pisces, Characidae). Heredity 69:50-56.

Silva DMZA, Pansonato-Alves JC, Utsunomia R, Araya-Jaime C, Ruiz-Ruano FJ, Daniel SN, Hashimoto DT, Oliveira C, Camacho JPM, Porto-Foresti F, et al. (2014) Delimiting the origin of a B chromosome by FISH mapping, chromosome painting and DNA sequence analysis in Astyanax paranae (Teleostei, Characiformes). PLoS One 9:e94896.

Silva DMZA, Daniel SN, Camacho JPM, Utsunomia R, Ruiz-Ruano FJ, Penitente M, Pansonato-Alves JC, Hashimoto DT, Oliveira
C, Porto-Foresti F, et al. (2016) Origin of B chromosomes in the genus Astyanax (Characiformes, Characidae) and the limits of chromosome painting. Mol Genet Genom 291:1407-1418.

Silva DMZA, Utsunomia R, Ruiz-Ruano FJ, Daniel SN, PortoForesti F, Hashimoto DT, Oliveira C, Camacho JPM and Foresti F (2017) High-throughput analysis unveils a highly shared satellite DNA library among three species of fish genus Astyanax. Sci Rep 7:12726.

Terán GE, Benitez MF and Mirande JM (2020) Opening the Trojan horse: phylogeny of Astyanax, two new genera and resurrection of Psalidodon (Teleostei: Characidae). Zool J Linnean Soc $\mathrm{XX}: 1-18$.

Torres-Mariano AR and Morelli S (2008). B chromosomes in a population of Astyanax eigenmanniorum (Characiformes, Characidae) from the Araguari River Basin (Uberlândia, MG, Brazil). Genet Mol Biol 31:246-249.

Utsunomia R, Ruiz-Ruano FJ, Silva DMZA, Serrano EA, Rosa IF, Scudeler PES, Hasimoto DT, Oliveria C, Camacho JPM and Foresti F (2017) A glimpse into the satellite DNA library in characidae fish (Teleostei, Characiformes). Front Genet 8:103.

Utsunomia R, Silva DMZA, Ruiz-Ruano FJ, Goes CAG, Melo S, Ramos LP, Oliveira C, Porto-Foresti F, Foresti F and Hashimoto DT (2019) Satellitome landscape analysis of Megaleporinus macrocephalus (Teleostei, Anostomidae) reveals intense accumulation of satellite sequences on the heteromorphic sex chromosome. Sci Rep 9:5856.

Vicari MR, Pistune HFM, Castro JP, Almeida MC, Bertollo LAC, Moreira-Filho O, Camacho JPM and Artoni RF (2011) New insights on the origin of B chromosomes in Astyanax scabripinnis obtained by chromosome painting and FISH. Genetica 139:1073-1081.

Associate Editor: Maria Jose de J. Silva

License information: This is an open-access article distributed under the terms of the Creative Commons Attribution License (type CC-BY), which permits unrestricted use, distribution and reproduction in any medium, provided the original article is properly cited. 